\title{
Tumor suppressor death-associated protein kinase 1 inhibits necroptosis by p38 MAPK activation
}

\author{
Yung-Hsuan Wu', Ting-Fang Chou', Leslie Young ${ }^{1}$, Fu-Yi Hsieh', Hsuan-Yin Pan', Shu-Ting Mo', Shani Bialik Brown², \\ Ruey-Hwa Chen ${ }^{3}$, Adi Kimchi ${ }^{2}$ and Ming-Zong Lai (i] ${ }^{1}$
}

\begin{abstract}
Death-associated protein kinase 1 (DAPK1, DAPk, DAPK) is known for its involvement in apoptosis and autophagyassociated cell death. Here, we identified an unexpected function of DAPK1 in suppressing necroptosis. DAPK1deficiency renders macrophages and dendritic cells susceptible to necroptotic death. We also observed an inhibitory role for DAPK1 in necroptosis in HT-29 cells, since knockdown or knockout of DAPK1 in such cells increased their sensitivity to necroptosis. Increased necroptosis was associated with enhanced formation of the RIPK1-RIPK3-MLKL complex in these DAPK1-deficient cells. We further found that DAPK1-deficiency led to decreased MAPK activated kinase 2 (MK2) activation and reduced RIPK1 S321 phosphorylation, with this latter representing a critical step controlling necrosome formation. Most TNF signaling pathways, including ERK, JNK, and AKT, were not regulated by DAPK. In contrast, DAPK bound p38 MAPK and selectively promoted p38 MAPK activation, resulting in enhanced MK2 phosphorylation. Our results reveal a novel role for DAPK1 in inhibiting necroptosis and illustrate an unexpected selectivity for DAPK1 in promoting p38 MAPK-MK2 activation. Importantly, our study suggests that modulation of necroptosis and p38/MK2-mediated inflammation may be achieved by targeting DAPK1.
\end{abstract}

\section{Introduction}

Necroptosis is typically induced in cells via activation of the receptor-interacting protein kinase 1 (RIPK1)-RIPK3 cascade and concomitant inactivation of the FADD-caspase-8-c-FLIP complex ${ }^{1-6}$. Necroptosis is triggered by a wide variety of stimuli including tumor necrosis factor (TNF), Toll-like receptor agonists, second mitochondria-derived activator of caspases (SMAC) mimetics, interferons (IFNs), microbial infection, or DNA-damaging chemicals ${ }^{7}$. Binding of TNF to TNF receptor 1 (TNFR1) leads to assembly of complex $\mathrm{I}^{8}$ at the plasma membranes by recruitment of TNF receptorassociated protein with death domain (TRADD) and RIPK1, followed by TNFR-associated factor 2 (TRAF2),

\footnotetext{
Correspondence: Ming-Zong Lai (mblai@gate.sinica.edu.tw)

${ }^{1}$ Institute of Molecular Biology, Academia Sinica, Taipei 11529, Taiwan

2Department of Molecular Genetics, Weizmann Institute of Science, Rehovot 76100, Israel

Full list of author information is available at the end of the article These authors contributed equally: Yung-Hsuan Wu, Ting-Fang Chou Edited by G. Raschellà
}

TRAF5, cellular inhibitors of apoptosis 1 (cIAP1) and cIAP2, and the linear ubiquitin chain assembly complex (LUBAC). The E3 ligases cIAP1/2 add K63 polyubiquitin chains to RIPK1 to recruit the transforming growth factor beta-activated kinase 1 (TAK1) and IкB kinase (IKK) complexes and induce NF- $\mathrm{kB}$ activation, whereas LUBAC introduces M1-linked polyubiquitin chains to RIPK1 and other complex I proteins for signaling. Loss of RIPK1 ubiquitination by depletion of cIAP $1 / 2$ or LUBAC or by deubiquitination through cylindromatosis protein (CYLD), together with association of FADD and caspase8 , result in complex II formation. Inactivation or depletion of caspase- 8 converts pro-apoptotic complex II into a pro-necroptotic complex, with RIPK1 binding and activating RIPK3 ${ }^{9-11}$, resulting in RIPK3 autophosphorylation and activation of mixed lineage kinase domain-like $(\mathrm{MLKL})^{12,13}$. Phosphorylated MLKL is translocated into the plasma membranes and wherein forms pores that lead to membrane leakage and necroptotic cell death ${ }^{14-16}$. Necroptosis associated with

\section{(c) The Author(s) 2020}

(c) (i) Open Access This article is licensed under a Creative Commons Attribution 4.0 International License, which permits use, sharing, adaptation, distribution and reproduction cc) in any medium or format, as long as you give appropriate credit to the original author(s) and the source, provide a link to the Creative Commons license, and indicate if changes were made. The images or other third party material in this article are included in the article's Creative Commons license, unless indicated otherwise in a credit line to the material. If material is not included in the article's Creative Commons license and your intended use is not permitted by statutory regulation or exceeds the permitted use, you will need to obtain permission directly from the copyright holder. To view a copy of this license, visit http://creativecommons.org/licenses/by/4.0/. 
depletion of FADD, caspase-8, or c-FLIP can be prevented by concomitant deletion of RIPK1 or RIPK $3^{17-19}$. In contrast, SMAC mimetics (used to promote cIAP1/2 degradation) and zVAD (a pan-caspase-inhibitor used to inactivate caspase-8) can induce necroptosis.

Recent studies have further revealed several cell death suppressing checkpoints centered on RIPK1. Complex Iassociated RIPK1 is subjected to phosphorylation by IKK that inhibits the transition into complex $\mathrm{II}^{20}$. In a different inhibitory process, TNF-induced activation of p38 MAPK leads to phosphorylation of MAPKAPK2 (MK2), with this latter directly phosphorylating RIPK1 at the S321 and S326 positions (in mouse; S320 and S325 in human) to prevent binding of RIPK1 with FADD/caspase- 8 to form complex $\mathrm{II}^{21-23}$. Alternatively, TBK1-mediated phosphorylation of RIPK1 inhibits RIPK1 activation and the conversion into complex $\mathrm{II}^{24,25}$.

Necroptosis participates in numerous pathological events $^{3-6,26}$. It often leads to inflammation and is one of the mechanisms involved in counteracting specific viral infections, while expression of viral molecules can result in evasion of the necroptotic process. Necroptosis also contributes to ischemia-reperfusion injury, transplantation rejection, and cancer inhibition or progressing ${ }^{3-6,26}$.

Death-associated protein kinase 1 (DAPK, DAPk, DAPK1) is a multi-domain serine/threonine kinase regulated by calcium ${ }^{27-29}$. It was first identified for its role in mediating IFN- $\gamma$-induced cell death ${ }^{30}$, but subsequent evidence demonstrated involvement in apoptotic cell death induced by $\mathrm{Fas}^{31}$, TGF- $\beta^{32}$, ceramide ${ }^{33}$, matrix detachment ${ }^{34}$, unliganded Netrin-1 receptor uncoordinated protein 5 homolog $2(\mathrm{UNC} 5 \mathrm{H} 2)^{35}$, or ER stress ${ }^{36}$. Although how DAPK1 regulates apoptosis remains incompletely understood ${ }^{37}$, it is known that it interacts with p53 and promotes p53-dependent cell death ${ }^{38,39}$. In addition, DAPK1 interacts with Fas-associated protein with death domain (FADD) ${ }^{38}$. DAPK1 is a tumor suppressor and is specifically downregulated in many types of cancer $^{40,41}$. Apart from its role in apoptosis, DAPK1 participates in a wide variety of cellular events including autophagy, membrane blebbing, and stress fiber formation that all contribute to its tumor-suppressing functions. In $\mathrm{T}$ lymphocytes, DAPK1 inhibits $\mathrm{T}$ cell activation by suppressing $\mathrm{T}$ cell receptor-induced NF- $\mathrm{kB}$ activation ${ }^{42}$.

In the present study, we found that DAPK1 negatively regulates necroptosis, unlike its active involvement in other forms of cell death. DAPK1-deficiency enhances the sensitivity of myeloid and HT-29 cells to necroptotic induction. Knockdown or knockout of DAPK1 in HT-29 cells increases their sensitivity to necroptosis. The increased necroptosis in DAPK1-deficient cells was associated with enhanced formation of the RIPK1-RIPK3-MLKL complex. We further found that DAPK1 selectively increased TNF-induced p38 MAPK and MK2 activation, leading to phosphorylation of RIPK1 at position S321 and inhibition of necrosome formation. Our results reveal a novel role for DAPK1 in inhibiting necroptosis and illustrate the diverse death-associated physiological functions regulated by DAPK1.

\section{Results \\ DAPK1-deficiency sensitizes myeloid cells to necroptosis}

We used bone marrow-derived macrophages (BMDMs) from wild-type control (WT) and $\mathrm{Dapk}^{-/-}$mice to examine the possible role of DAPK1 in necroptosis. DAPK1 knockout did not affect the development of myeloid cells in bone marrow or spleen (Supplementary Fig. 1), nor did DAPK1 deficiency affect the protein expression of RIPK1, RIPK3, MLKL, or FADD in BMDMs (Fig. 1a). Treatment of BMDMs with the SMAC mimetic AT-406 or the pan-caspase inhibitor zVAD alone did not affect macrophage viability, as measured by release of ATP (Fig. 1b, c). However, a combination of zVAD and AT-406 induced cell death in BMDMs, which was suppressed by the inclusion of RIPK1 inhibitor necrostatin-1 (Nec-1), confirming its necroptotic nature (Fig. 1b). Unexpectedly, DAPK1-deficient BMDMs were much more sensitive to cell death induced by zVAD plus AT406 than WT BMDMs (Fig. 1b). We observed a similar necroptotic outcome in Dapk $1^{-1-}$ BMDMs when we used zVAD together with another SMAC mimetic, BV6 (Fig. 1c). In addition, DAPK1-deficient macrophages exhibited higher sensitivity to necroptotic death triggered by zVAD plus TNF or zVAD plus IFN- $\beta^{7}$ (Fig. 1d, e). We also tested the sensitivity of Dapk1 $1^{-1-}$ BMDMs to SMAC mimetic alone in the absence of zVAD. At higher dose $(5 \mu \mathrm{M})$, AT406 triggered necroptosis which was significantly enhanced by DAPK1 deficiency (Fig. 1f). We also measured cell viability according to incorporation of MTT (3(4,5-dimethylthiazol-2-yl)-2,5-diphenyl tetrazolium bromide) (Supplementary Fig. 2), which showed that treatments with zVAD+AT-406 lowered the viability of Dapk1 $1^{-1}$ BMDMs compared to WT BMDMs, but addition of Nec-1 effectively restored cell viability. We observed similar findings in bone marrow-derived dendritic cells (Supplementary Fig. 3).

We then knocked down DAPK1 in the mouse macrophage cell line J774A.1 (Supplementary Fig. 4a). By itself, zVAD $(20 \mu \mathrm{M})$ triggered weak necroptosis in WT J774A.1 cells (Supplementary Fig. 4b), but this effect was markedly enhanced upon DAPK1 knockdown, and Nec-1 addition completely attenuated the impact of DAPK1 knockdown (Supplementary Fig. 4b). We could induce significant cell death in DAPK1-knockdown J774A.1 cells via a combination of a low concentration of zVAD $(5 \mu \mathrm{M})$ plus AT-406 (Supplementary Fig. 4c). Furthermore, reintroduction of DAPK1 to DAPK1-deficient J774A.1 cells rescued them from the necroptosis triggered by 

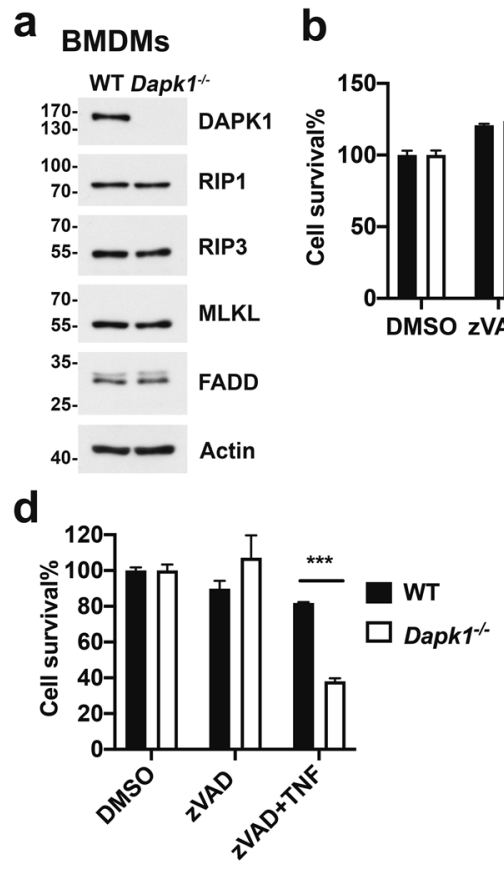

b

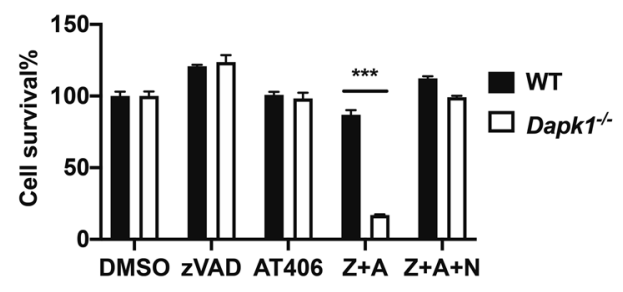

e

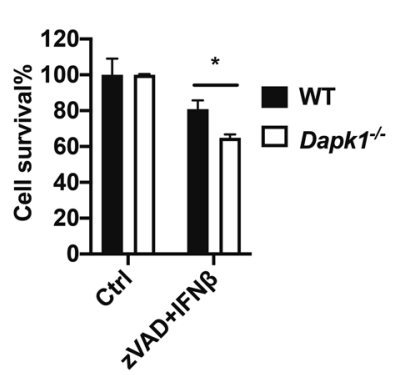

C

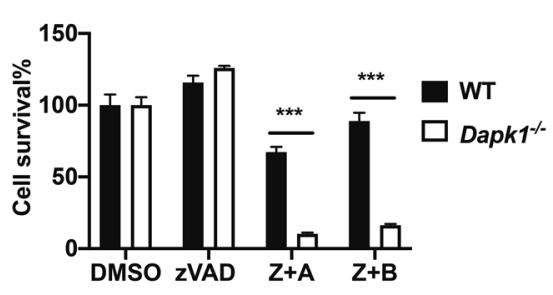

f

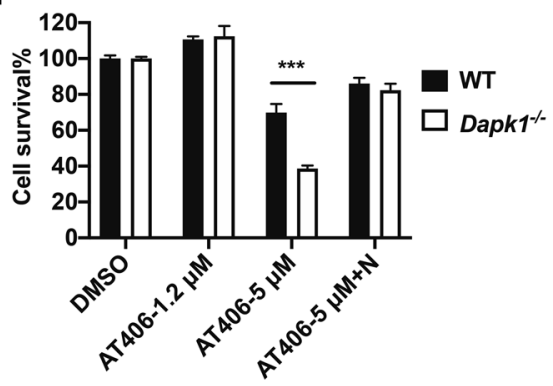

Fig. 1 DAPK1-deficient BMDMs are more sensitive to necroptotic induction. a DAPK1 deficiency does not affect expressions of FADD, RIPK1, RIPK3, or MLKL in BMDMs. b, $\mathbf{c}$ Dapk1 ${ }^{-1-}$ BMDMs exhibit increased cell death induction relative to WT upon zVAD+AT-406 treatment. WT and Dapk1 ${ }^{-1-}$ BMDMs were stimulated with DMSO, AT-406 $(0.6 \mu \mathrm{M}, \mathrm{A}), \mathrm{ZVAD}(20 \mu \mathrm{M}, \mathrm{Z}), \mathrm{Nec}-1(40 \mu \mathrm{M}, \mathrm{N})$, or BV6 $(0.5 \mu \mathrm{M}, \mathrm{B})$, as indicated, for $18-20 \mathrm{~h}$, before determining cell death according to release of ATP. $\mathbf{d}$, e zVAD+TNF or zVAD+IFN- $\beta$ treatments trigger increased necroptosis in Dapk $1^{-/-}$ BMDMs. WT and Dapk $1^{-/-}$BMDMs were treated with zVAD + TNF $(5 \mathrm{ng} / \mathrm{ml})(\mathbf{d})$ or ZVAD + IFN- $\beta(5 \mathrm{ng} / \mathrm{ml})(\mathbf{e})$ and then cell viability was determined. f High dose of AT-406 induces necroptosis. WT and Dapk1 ${ }^{-1-}$ BMDMs were treated with AT-406 at the indicated dose, without or with Nec-1, and cell viability quantitated. Values are mean $\pm \mathrm{SD}$ of triplicates in a single experiment. ${ }^{*} P<0.05,{ }^{* *} P<0.01,{ }^{* * *} P<0.001$ for unpaired $t$-test. Data have been repeated in two $(\mathbf{e}, \mathbf{f})$ or three $(\mathbf{a}-\mathbf{d})$ independent experiments.

zVAD+AT-406 (Supplementary Fig. 4c), confirming the specific involvement of DAPK1 in this process ${ }^{43}$.

In contrast, we found that Dapk1 ${ }^{-1-}$ BMDMs were more resistant to thapsigargin-triggered apoptosis than WT BMDMs (Supplementary Fig. 5a), consistent with the pro-apoptotic role of DAPK1 in ER stress-induced cell death ${ }^{36}$. In Jurkat cells, a cell line sensitive to Fasinitiated apoptosis, DAPK1 knockdown did not affect surface Fas expression but it did reduce Fas ligand (FasL)-triggered cell death (Supplementary Fig. 5b, c). BMDMs are moderately sensitive to FasL-induced apoptosis, and we found that DAPK1-deficiency reduced the extent of cell death mediated by FasL in such cells (Supplementary Fig. 5d). Therefore, consistent with the known involvement of DAPK1 in apoptosis, DAPK1-deficiency attenuates ER stress- and FasLinduced cell death. The enhanced susceptibility of DAPK1-deficient myeloid cells to necroptosis reveals a selective inhibitory role for DAPK1 in necroptosis.

\section{Necroptosis is increased upon downregulation of DAPK1 in} HT-29 cells

The enhanced sensitivity to necroptosis was not restricted to Dapk1 ${ }^{-/-}$myeloid cells. A similar effect was found in the human colon adenocarcinoma cell line HT29 . HT-29 cells were previously shown to be susceptible to necroptotic induction by treatment with zVAD plus SMAC mimetics ${ }^{9}$. We knocked down DAPK1 by shRNA in HT-29 cells, which did not affect expression of RIPK1 or RIPK3 (Fig. 2a). Treatment of WT HT-29 cells with zVAD or BV6 alone did not trigger cell death, as measured by propidium iodide (PI) staining (Fig. 2b). However, a combination of zVAD plus BV6 did induce cell death in WT HT-29 cells, and this outcome was significantly enhanced upon DAPK1 deficiency (Fig. 2b). The necroptotic nature of the cell death outcome was confirmed by addition of Nec-1, which suppressed the effect in both WT and DAPK1-knockdown HT-29 cells (Fig. 2b).

We also generated DAPK1-knockout HT-29 cells by CRISPR-Cas9 editing (Supplementary Fig. 6a) and found that expression of necroptosis-associated molecules (RIPK1, RIPK3, MLKL, and FADD) was comparable between WT and DAPK1-knockout HT-29 cells (Supplementary Fig. 6b). The necroptosis induced by zVAD+BV6 was also significantly enhanced in these DAPK1-knockout HT-29 cells and, importantly, it was inhibited by Nec-1 addition (Supplementary Fig. 6c). 


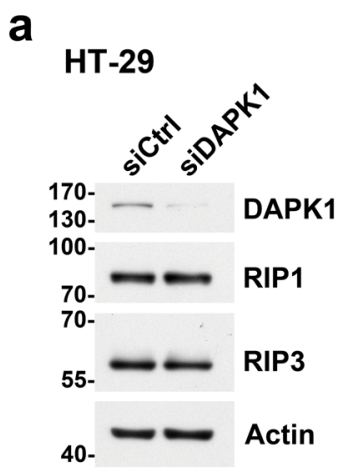

C

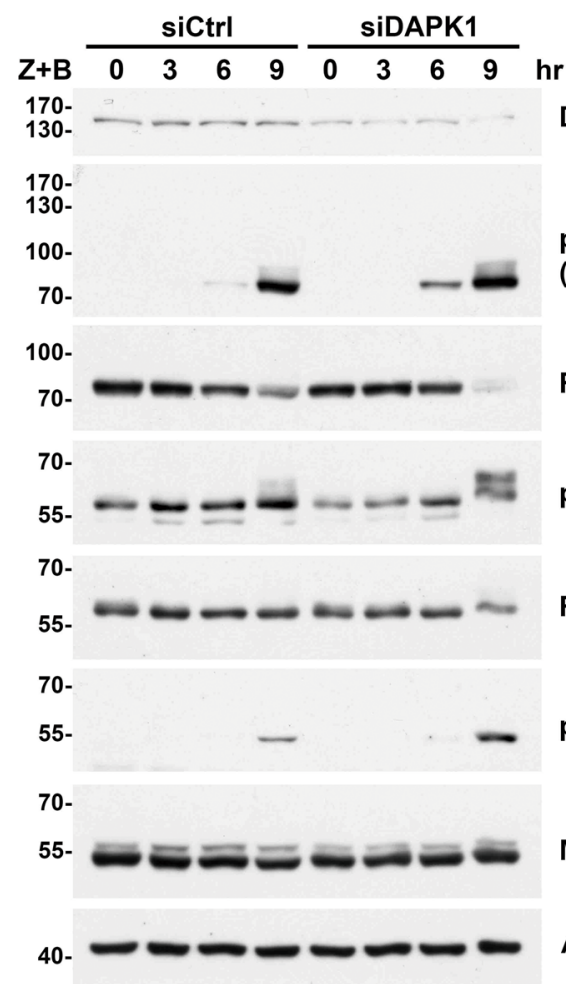

b

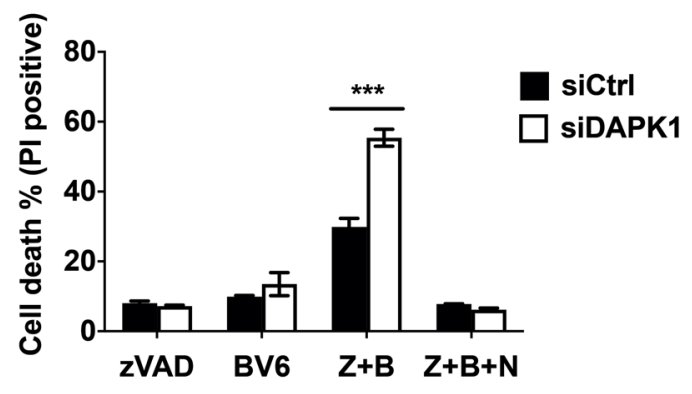

DAPK1

d

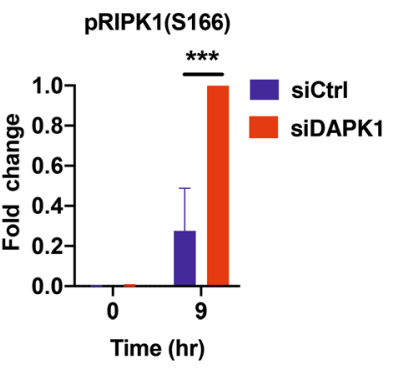

RIPK1

pRIPK3

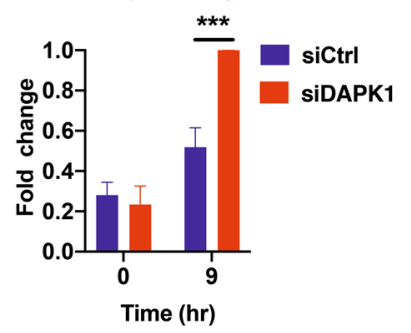

PMLKL

MLKL

Actin

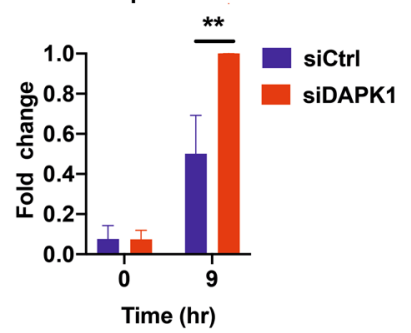

Fig. 2 Knockdown of DAPK1 confers increased sensitivity to necroptosis on HT-29 cells. a Knockdown of DAPK1 in HT-29 cells. HT-29 cells were transduced with pLL3.7-shCtrl or pLL3.7-shDAPK1, sorted, and then expressions of DAPK1, RIPK1 and RIPK3 were determined. $\mathbf{b}$ Increased zVAD

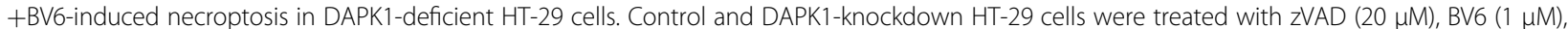
Nec-1 $(40 \mu \mathrm{M})$ as indicated for $24 \mathrm{~h}$, cell death was quantitated by PI staining. Values are mean \pm SD of triplicates in a single experiment. ${ }^{* * *} P<0.001$ for unpaired $t$-test. Results have been confirmed in three independent experiments. $\mathbf{c}$ Enhanced zVAD+BV6-induced phosphorylation of RIPK1, RIPK3 and MLKL in DAPK1-deficient HT-29 cells. Control and DAPK1-knockdown HT-29 cells were treated with ZVAD+BV6 $(0.5 \mu M, Z+B)$ and contents of RIPK1, pS166-RIPK1, RIPK3, pS227-RIPK3, MLKL, and pMLKL in cell lysates were determined at the indicated time points. Right panel, quantitation of pRIPK1(S166), pRIPK3 and pMLKL from three independent experiments using normalized intensity of pRIPK1(S166), pRIPK3 and pMLKL in DAPK1knockdown HT29 cells at $9 \mathrm{~h}$ as $1 .{ }^{* *} P<0.01,{ }^{* * *} P<0.001$ for two-way ANOVA followed by a Tukey's multiple comparison test.

Therefore, DAPK1-deficiency enhances necroptotic induction in macrophages, dendritic cells and HT-29 cells, supporting the inhibitory role of DAPK1 in necroptosis.

\section{Overexpression of DAPK1 inhibits necroptosis}

We next generated HT-29 cells that overexpress DAPK1 (Supplementary Fig. 7a). The necroptosis induced by zVAD plus TNF treatment was attenuated in 


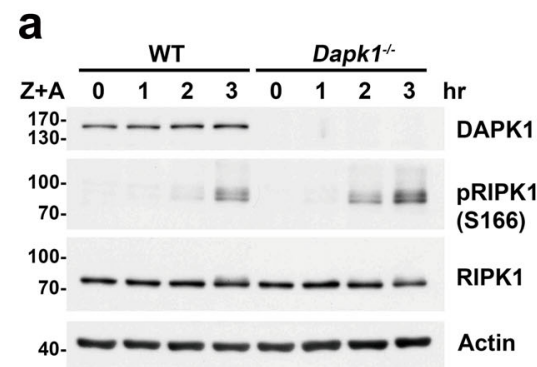

b

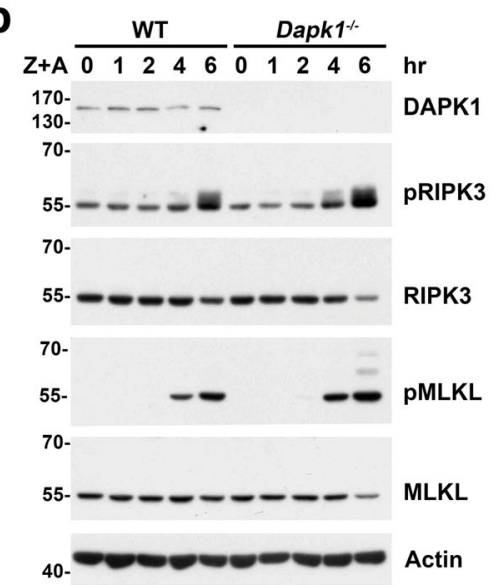

C

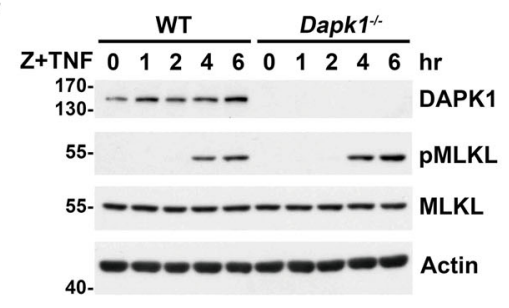

d

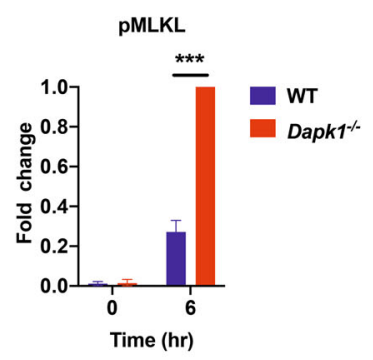

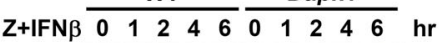

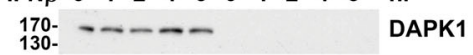

55- $\quad \ldots \quad \ldots$ pMLKL

55- $-0-0-0-0-0-3 L K$

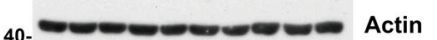

Fig. 3 Enhanced activation of RIPK1, RIPK3 and MLKL in DAPK1-deficient cells after necroptotic induction. $\mathbf{a}$, $\mathbf{b}$ Increased $Z$ VAD $+A T-406-$

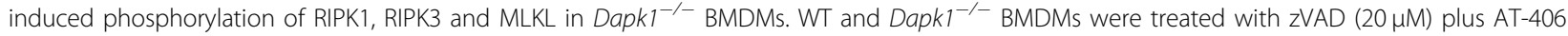
$(0.6 \mu \mathrm{M}, \mathrm{Z}+\mathrm{A})$, and cell lysates were prepared at the indicated time points. The contents of RIPK1, pS166-RIPK1 (a), or RIPK3, PRIPK3, MLKL, and pMLKL (b) were determined by Western blot. Right panel, quantitation of pRIPK1(S166) (a) and pMLKL (b) from three independent experiments using normalized intensity of pRIPK1(S166) (3 h) and pMLKL (6 h) in Dapk $1^{-1-}$ BMDMs as $1 .{ }^{* * *} P 0.001$ for two-way ANOVA followed by a Tukey's multiple comparison test. $\mathbf{c}$, $\mathbf{d}$ Increased activation of MLKL in Dapk1 $1^{-1-}$ BMDMs stimulated by treatment with zVAD plus TNF or IFN- $\beta$. WT and Dapk $1^{-/-}$ BMDMs were treated with zVAD in combination with TNF (c), or IFN- $\beta$ (d) and levels of MLKL and pMLKL in cell lysates were determined at the indicated time points. Data are representative of three independent experiments.

DAPK1-overexpressing HT-29 cells relative to vector control (Supplementary Fig. 7b). We were also able to recapitulate the anti-necroptotic effect of DAPK1 by reintroducing it into DAPK1-null HT-29 cells (Supplementary Fig. 7c, d). Together, these results further validate that DAPK1 suppresses necroptosis.

\section{Increased phosphorylation of RIPK1(S166), RIPK3, and MLKL in DAPK1-deficient cells}

Apart from assessing cell death and viability, we also examined certain biochemical events associated with necroptosis in our Dapk $1^{-1-}$ cells. During TNF-initiated necroptotic signaling, RIPK1 is first phosphorylated at S166, before the RIPK1-mediated activation of RIPK3 that induces MLKL activation and cell death. Treatment of BMDMs with zVAD+AT-406 induced activation of RIPK1, RIPK3, and MLKL (Fig. 3a, b). We also observed increased phosphorylation of RIPK1(S166), RIPK3 and MLKL in Dapk1 $1^{-1-}$ BMDMs relative to WT BMDMs upon treatment with zVAD+AT-406 (Fig. 3a, b).
Moreover, we detected enhanced MLKL phosphorylation in Dapk1 $1^{-1-}$ BMDMs upon treatment with zVAD plus either TNF or IFN- $\beta$ (Fig. 3c, d).

Similarly, RIPK1(S166), RIPK3, and MLKL all displayed increased phosphorylation in both DAPK1-knockdown and DAPK1-knockout HT-29 cells (relative to WT HT-29 cells) after zVAD+BV6 combinatory treatment (Fig. 2c, d, Supplementary Fig. 6d). Therefore, DAPK1-deficient cells exhibit enhanced activation of the RIPK1-RIPK3-MLKL axis, which is consistent with their increased necroptotic activity.

\section{Increased association of RIPK1 with FADD and caspase-8 in DAPK1-deficient cells}

TNF-induced necroptosome formation begins upon binding of RIPK1 to FADD and caspase-8. We observed that immunoprecipitation of FADD brought down RIPK1 and RIPK1 phosphorylated at position S166 [pRIPK1 (S166)] after BMDMs had been treated with zVAD+AT406 (Fig. 4). However, DAPK1-deficiency increased the 

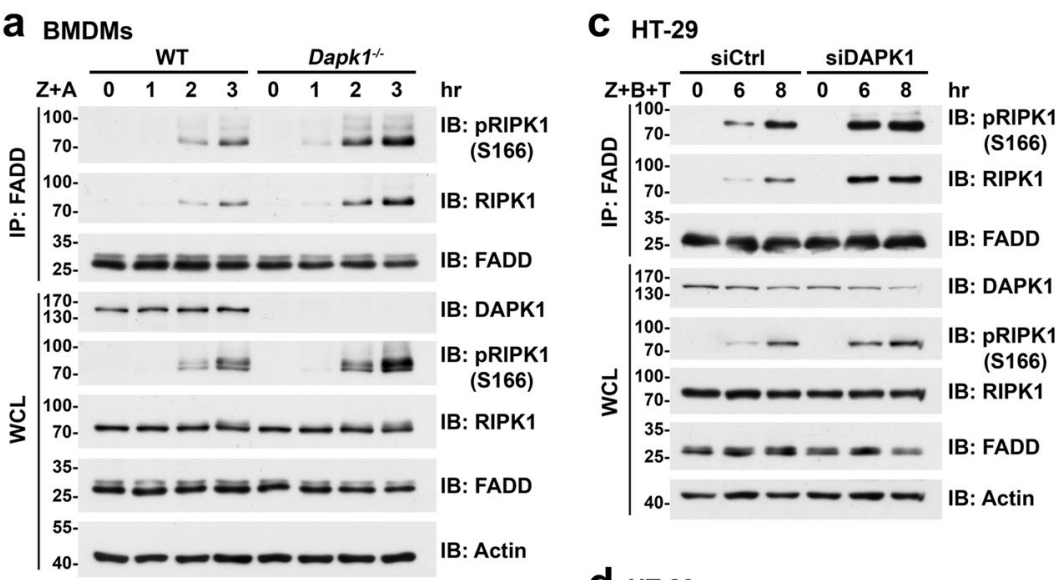

\section{b BMDMs}

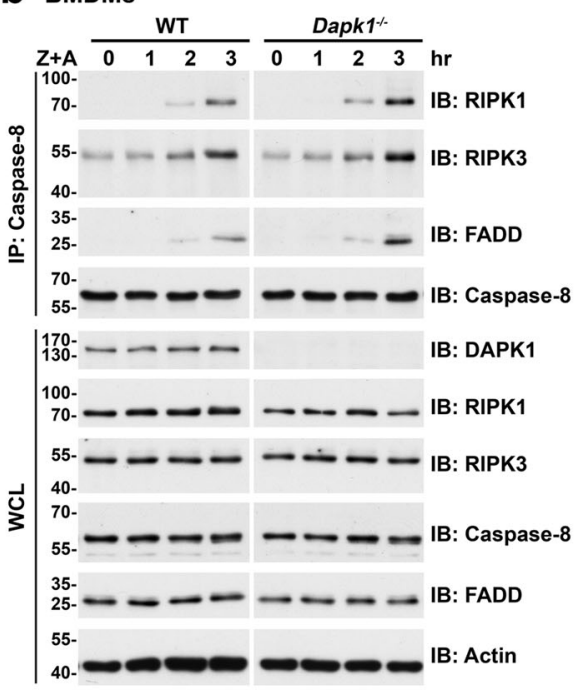

\section{d HT-29}

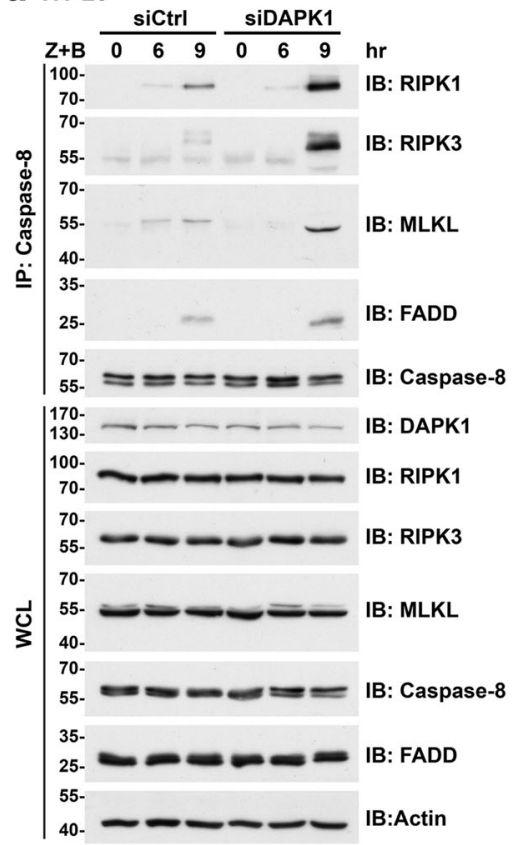

Fig. 4 Enhanced associations of RIPK1 and RIPK3 with FADD or caspase-8 in DAPK1-deficient cells. $\mathbf{a}$, b Enhanced zVAD+AT-406-induced binding of RIPK1 and RIPK3 to FADD/caspase-8 in Dapk1 ${ }^{-1}$ BMDMs. WT and Dapk $1^{-1}$ BMDMs were treated with zVAD+AT-406 (Z + A), and wholecell lysates (WCL) were prepared at the indicated time points. WCL were immunoprecipitated with anti-FADD and contents of pRIPK1, RIPK1 and FADD in the precipitates and WCL were determined (a), or they were immunoprecipitated with anti-caspase-8 and the amounts of RIPK1, RIPK3, FADD and caspase-8 in the precipitates and WCL were determined (b). $\mathbf{c}$, $\mathbf{d}$ Increased binding of RIPK1 and RIPK3 to FADD/caspase-8 in DAPK1deficient HT-29 cells upon necroptotic induction. Control and DAPK1-knockdown HT-29 cells were treated with zVAD+BV6 + TNF $(Z+B+T)(\mathbf{c})$ or zVAD+BV6 $(Z+B)(\mathbf{d})$ before collecting WCL at the indicated time points. WCL were immunoprecipitated with anti-FADD and the contents of PRIPK1, RIPK1 and FADD in the precipitates and WCL were determined (c), or we immunoprecipitated them with anti-caspse-8 and the amounts of RIPK1, RIPK3, FADD and caspase-8 were determined in the precipitates and WCL (d). Data are representative of three independent experiments.

association of both RIPK1 and pRIPK1(S166) with FADD upon zVAD+AT-406 stimulation (Fig. 4a). Similarly, immunoprecipitation of caspase-8 pulled down RIPK1, RIPK3 and FADD in macrophages treated with zVAD +AT-406, and levels of caspase-8-bound RIPK1 and RIPK3 were higher in Dapk1 $1^{-1-}$ BMDMs than WT counterparts after necroptosis induction (Fig. 4b).

We observed a comparable scenario for DAPK1-deficient HT-29 cells. Treatment of HT-29 cells with zVAD plus both BV6 and TNF led to stronger association of FADD with RIPK1 and pRIPK1(S166) (Fig. 4c). DAPK1knockdown increased the binding of RIPK1 and pRIPK1 (S166) to FADD. Immunoprecipitation of caspase-8 also brought down more RIPK1, RIPK3, MLKL, and FADD from DAPK1-deficient HT-29 cells than was the case for control HT-29 cells (Fig. 4d). Together, these results illustrate that DAPK1-deficiency enhances formation of the FADD-caspase-8-RIPK1-RIPK3-MLKL complex. 


\section{Sensitization to TNF-induced septic shock in DAPK1- knockout mice}

We also examined whether DAPK1-deficiency increases necroptosis in vivo. In RIPK1- and RIPK3-dependent processes, necroptosis mediates TNF-induced systemic inflammatory response syndrome. The administration of TNF-induced hypothermia but did not trigger death in WT mice (Fig. 5a, b). DAPK1-deficient mice were highly sensitive to severe hypothermia and lethality triggered by TNF (Fig. 5a, b). Therefore, DAPK1-deficiency conferred sensitivity to necroptosis in cultured cells and in vivo.

\section{DAPK1-deficiency does not affect major TNF-induced survival signaling}

We next examined how DAPK1 inhibits TNF-mediated necroptosis by determining TNF-induced survival signaling in WT and Dapk1 $1^{-1-}$ BMDMs. TNF-triggered $\mathrm{I} \kappa \mathrm{B} \alpha$ phosphorylation and $\mathrm{I} \kappa \mathrm{B} \alpha$ degradation was comparable between WT and Dapk1 $1^{-1-}$ BMDMs (Fig. 5c). Similarly, activation of extracellular signal-regulated kinase (ERK) and JNK by TNF was practically indistinguishable between WT and Dapk1 $1^{-1-}$ BMDMs. TNF also triggered similarly moderate AKT activation in both WT and Dapk1 $1^{-1-}$ BMDMs (Fig. 5c). We also assessed if DAPK1 affected degradation of cIAP1 by the SMAC mimetics AT-406 and BV6. AT-406-mediated cIAP1 downregulation was comparable between WT and Dapk $1^{-1-}$ BMDMs (Supplementary Fig. 8a), and DAPK1knockdown did not affect cIAP1-degradation triggered by BV6 in HT-29 cells (Supplementary Fig. 8b). These results suggest that DAPK1 deficiency does not affect most TNFinduced survival signals nor alter cIAP-1 degradation.

\section{Enhanced necroptosis in DAPK1-deficient cells is associated with decreased MK2-directed S321 phosphorylation of RIPK1}

Despite that the majority of TNF-induced survival signaling were not affected by DAPK1-deficiency (Fig. 5c), recent studies have revealed that kinases downstream to TNFR can regulate RIPK1-containing death complex assembly in unconventional ways. For instance, IKK $\alpha / \mathrm{IKK} \beta$ inhibits RIPK1-mediated death independently of NF-kB activation via direct phosphorylation of RIPK $1^{20}$. Moreover, p38/MK2-mediated phosphorylation of RIPK1 at positions S321 and S326 inhibits binding of RIPK1 to FADD/caspase$8^{21-23}$. Accordingly, we examined activation of IKK $\alpha / \mathrm{IKK} \beta$, p38 MAPK and MK2, as well as phosphorylation of RIPK1 at S321, in DAPK1-deficient macrophages following TNF stimulation. We found that TNF-triggered activation of $\mathrm{IKK} \alpha / \mathrm{IKK} \beta$ was comparable between WT and Dapk1 $1^{-/-}$ BMDMs (Fig. 5d), but we did find reduced activation of $\mathrm{p} 38$ MAPK and decreased S321 phosphorylation of RIPK1 in Dapk $1^{-1-}$ BMDMs relative to WT (Fig. $5 \mathrm{~d}$, e). The reduced phosphorylation of p38 MAPK in Dapk $1^{-1-}$ BMDMs was also visualized in image analysis (Supplementary Fig. 9). Consistent with attenuated phosphorylation of p38 MAPK and RIPK1(S321) in DAPK-deficient cells, MK2 activation was compromised in DAPK-deficient macrophages (Fig. 5f). These outcomes likely reflect the enhanced formation of FADD-caspase-8-RIPK1-RIPK3 complex in DAPK1deficient cells (Fig. 4). In WT BMDMs, treatment with p38 MAPK inhibitor SB203580 or MK2 inhibitor PF3644022 sensitized BMDMs to necroptosis induced by zVAD+AT406 (Fig. 5g), to the levels observed in Dapk $1^{-/-}$ BMDMs (Fig. 1). Therefore, the increased necroptosis observed in DAPK1-deficient cells could be partly attributable to decreased activation of p38 MAPK and MK2, as well as attenuated RIPK1 S321 phosphorylation.

RIPK1 S321 phosphorylation also regulates RIPK1induced apoptosis ${ }^{21-23}$. We determined whether TNFinduced apoptosis was affected by DAPK1 deficiency. Consistent with the inhibitory role of DAPK1 in RIPK1mediated necroptosis, DAPK1 deficiency also conferred sensitivity to RIPK1-mediated apoptosis induced by TNF and AT-406 (Supplementary Fig. 10a). Suppression of either p38 MAPK or MK2 sensitized WT macrophages to RIPK1-mediated apoptosis (Supplementary Fig. 10b). Together, DAPK1-induced activation of p38 MAPK and MK2, leading to RIPK1 S321 phosphorylation, prevents TNF-triggered necroptosis and apoptosis.

\section{DAPK1 binds $\mathrm{p} 38$ MAPK and promotes $\mathrm{p} 38$ activation}

MK2 is activated by 38 MAPK in the nucleus, followed by cytoplasmic entry to phosphorylate target substrates $^{44,45}$. We determined the distribution of MK2 in Dapk $1^{-/-}$macrophages before and after TNF stimulation. The overall quantity of MK2 in the cytosol and nucleus was comparable between WT and Dapk $1^{-/-}$macrophages (Supplementary Fig. 11). The reduced phosphorylation of MK2 in Dapk1 $1^{-/-}$macrophages was thus not associated with altered cytoplasmic presence of MK2.

We investigated how DAPK1 affects p38 MAPK activation. TNF-induced MKK3 phosphorylation was comparable between WT and Dapk1 ${ }^{-1-}$ BMDMs (Fig. 6a), the attenuated TNF-triggered p38 MAPK activation in Dapk $1^{-1-}$ BMDMs therefore suggest that p38 MAPK is the stage downstream of TNFR which is regulated by DAPK1.

We found that the kinase activity of DAPK1 was not involved in necroptosis inhibition. The introduction of kinase-dead DAPK1 mutant (DAPK1[K42A]) was as effective as WT DAPK1 in enhancing the survival of DAPK1-null HT-29 cells to necroptosis (Fig. 6b), as well as increasing the activation of p38 MAPK (Fig. 6c). Collectively, the kinase activity of DAPK1 is not required for conferring the resistance to necroptosis.

Introduction of p38 MAPK into DAPK $1^{-/-} \mathrm{HT}-29$ cells increased the resistance to necroptosis induction (Fig. 6d). 


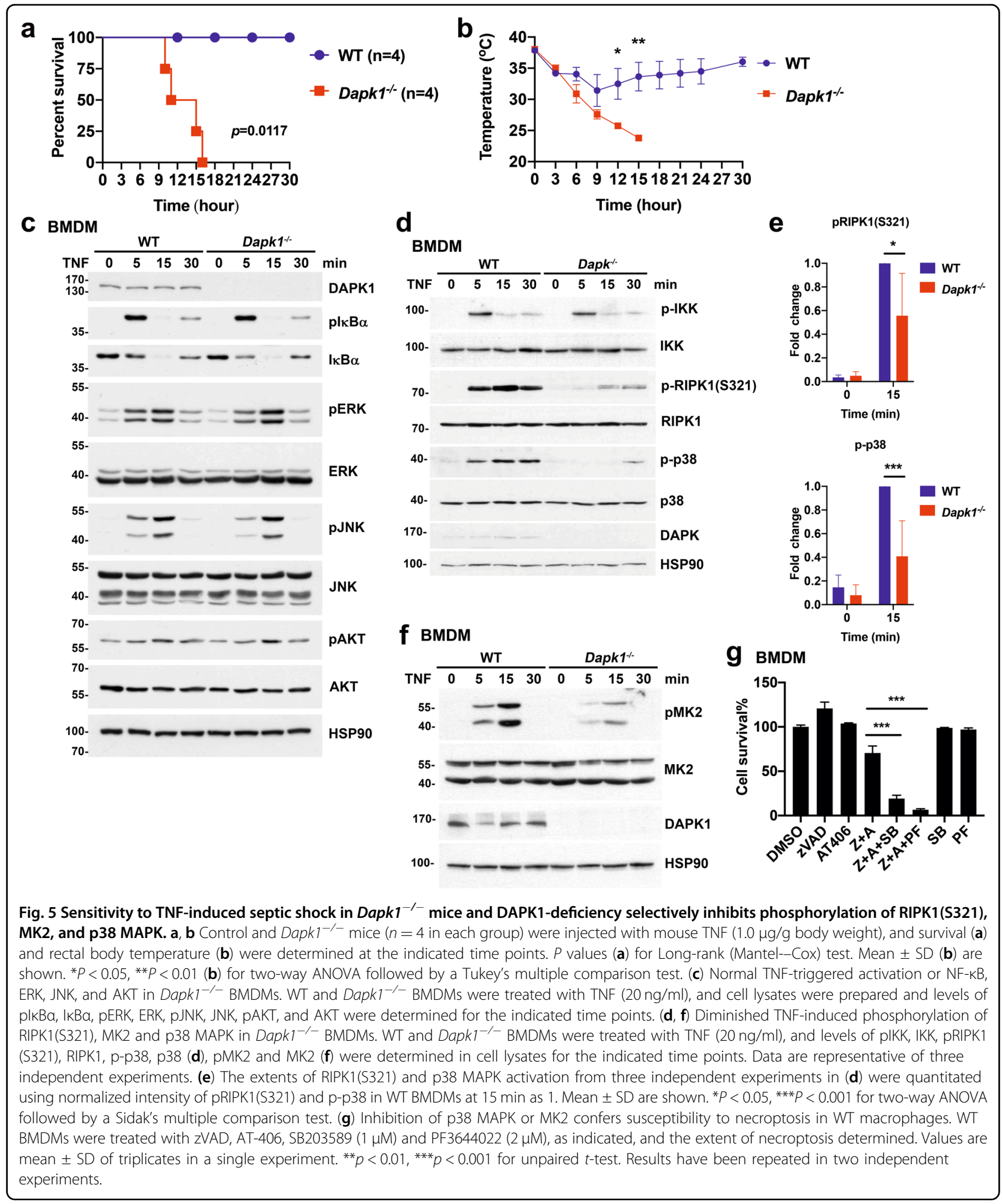

Overexpression of MKK3 alone, however, did not increase the survival of DAPK1-null HT-29 cells to necroptosis. In addition, DAPK1 was found to interact with $\mathrm{p} 38$ MAPK in HEK293T cells, in which p38 MAPK-HA was pulled down by DAPK1-FLAG, and vice versa (Fig. 6e). Recombinant p38 MAPK also bound purified DAPK1 protein (Fig. 6f), suggesting that DAPK1 may affect p38 MAPK activation through binding p38 MAPK. This was 


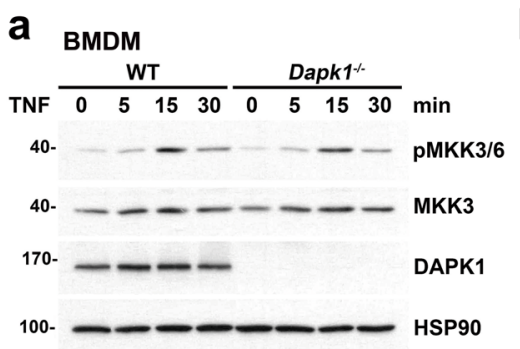

b

d
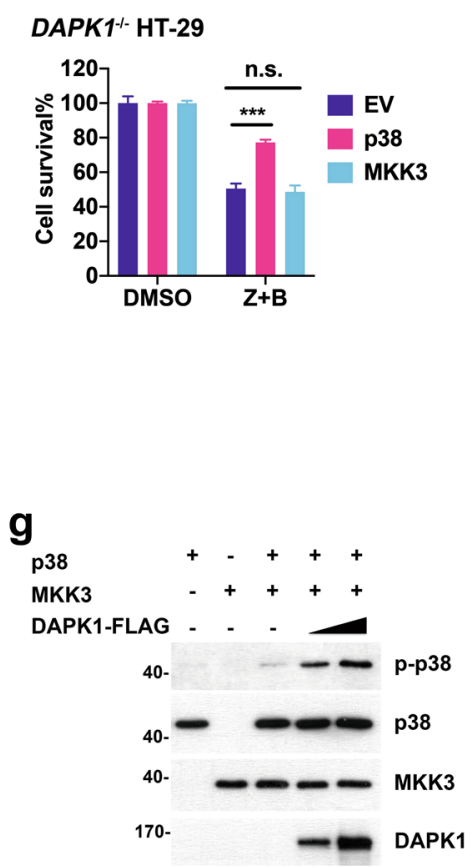

h

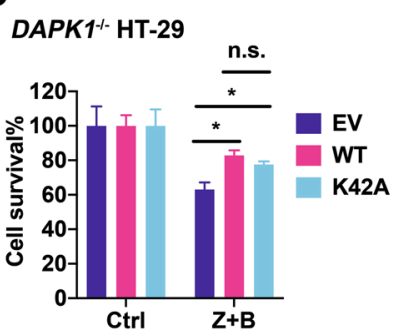

C HEK293T

DAPK1-FLAG + - +

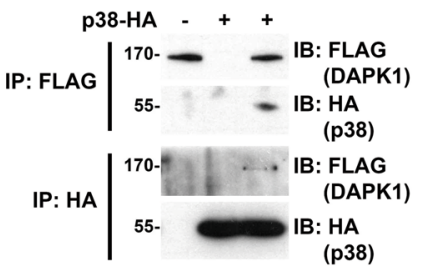

(p38)
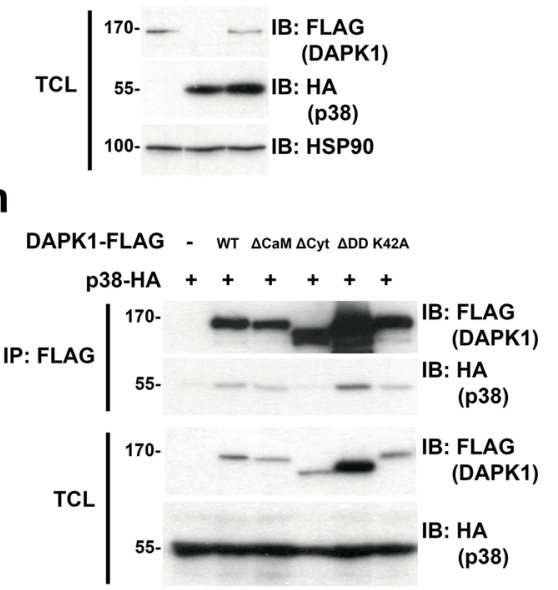

C

DAPK1 ${ }^{-1-}$ HT-29

TNF $\frac{\text { Mock }}{07.51530} \frac{\text { WT }}{07.51530} \frac{\text { K42A }}{07.51530 \mathrm{~min}}$

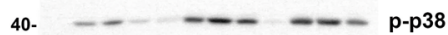

40. - p38

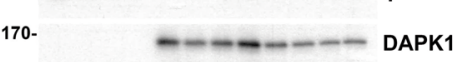

100- $-1-1-1-1-1-$ HSP9O

f

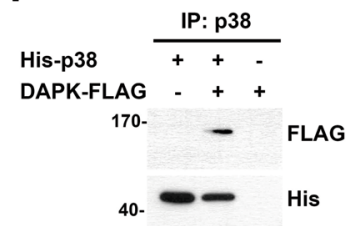

i

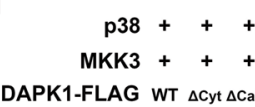

DAPK1-FLAG WT $\triangle$ Cyt $\triangle \mathrm{CaM}$
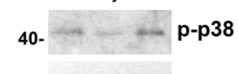

40- $--\mathrm{p} 38$

40-

$170-$

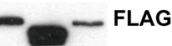

Fig. 6 DAPK1-deficiency selectively inhibits phosphorylation of RIPK1(S321), MK2, and p38 MAPK. a DAPK1-deficiency does not affect MKK3 activation. WT and Dapk1 ${ }^{-1-}$ BMDMs were treated with TNF, and levels of pMKK3 and MKK3 were determined in cell lysates. b DAPK1 [K42A] increases resistance to necroptosis in DAPK1-deficient cells. DAPK1 ${ }^{-1-}$ HT-29 cells were transfected with empty vector (EV), or transfected with WT DAPK1 or DAPK1 [K42A]. Cells were treated with zVAD (Ctrl) or zVAD + BV6, and viability determined by ATP assay. $\mathbf{c}$ Either WT DAPK or DAPK1[K42A] increases $\mathrm{p} 38$ MAPK activation in DAPK1-null cells. DAPK1 ${ }^{-1-}$ HT-29 cells, mock transduced, transduced with WT DAPK1 or DAPK1 [K42A] were treated with TNF $(20 \mathrm{ng} / \mathrm{ml})$ for the indicated time points, and the levels of DAPK1, p38 MAPK and phospho-p38 MAPK were determined. $\mathbf{d}$ Overexpression of p38 MAPK inhibits necroptosis. DAPK1 ${ }^{-1-}$ HT-29 cells were transfected with empty vector (EV), p38 MAPK or MKK3. The extent of necroptosis induced by zVAD+BV6 was determined by ATP release. e Interaction between DAPK1 and p38 MAPK. HEK293T cells were transfected with DAPK1FLAG and/or p38-HA as indicated. Cell lysates were prepared 24 later, and precipitated with anti-FLAG or anti-HA. f p38 MAPK binds DAPK in vitro. Recombinant human p38 MAPK (200 ng) was incubated with purified DAPK (100 ng), as indicated. p38 MAPK was pulled down by anti-p38 MAPK (ab170099, Abcam), and the presence of DAPK and p38 MAPK in immunoprecipitants determined by anti-FLAG and anti-His, respectively. Results have been independently confirmed using another anti-p38 MAPK (7218, Cell Signaling). g DAPK1 promotes MKK3-directed p38 MAPK phosphorylation. Recombinant p38 MAPK (50 ng), MKK3 (100 ng), and DAPK1-FLAG (25 ng or $75 \mathrm{ng}$ ) was incubated as indicated in ATP-containing kinase buffer at $30^{\circ} \mathrm{C}$ for $1 \mathrm{~h}$. The amounts of p38 MAPK, MKK3, DAPK1 and phospho-p38 MAPK was determined by immunoblots. $\mathbf{h}$ Binding of different DAPK1 mutants to p38 MAPK. HEK293T cells were transfected with different mutants of DAPK1-FLAG and p38-HA as indicated. The presence

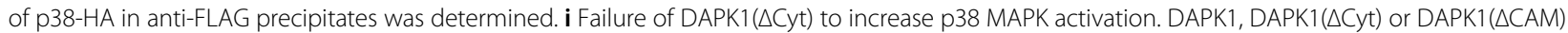
was evaluated for its ability to increase MKK3-directed p38 MAPK activation described in ( $\mathbf{g}$ ). Values are mean \pm SD of triplicates in a single experiment. ${ }^{*} P<0.05,{ }^{* * *} P<0.001$ (b, d) for two-way ANOVA followed by a Tukey's multiple comparison test. Results have been repeated in three $(\mathbf{a}-\mathbf{c}, \mathbf{e}, \mathbf{f})$ or two $(\mathbf{d}, \mathbf{g}-\mathbf{i})$ independent experiments.

confirmed in an in vitro kinase analysis, in which recombinant MKK3 was used to activate recombinant $\mathrm{p} 38$ MAPK. The addition of increasing amounts of recombinant DAPK1 increased MKK3-directed p38 MAPK phosphorylation (Fig. 6g). In contrast, DAPK1 $[\Delta \mathrm{Cyt}]$, the DAPK1 mutant lacking cytoskeleton- 
interacting domain, failed to bind p38 MAPK (Fig. 6h), and was less effective to activate p38 MAPK than WT DAPK1 (Fig. 6i). Therefore, DAPK1 selectively increases p38 MAPK activation likely by its specific interaction with p38 MAPK.

Together, our results illustrate that DAPK1 inhibits necroptosis induction through enhanced activation of p38-MK2-RIPK1 cascade. DAPK1 specifically targets the activation of p38 MAPK by binding p38 MAPK, with consequently increased activation of MK2 and elevated RIPK1 S321 phosphorylation, resulting in suppression of necroptosis.

\section{Discussion}

In this study, we identified an unexpected role for DAPK1 in necroptosis. DAPK1 has mostly been shown to promote cell death. It mediates the apoptotic cell death induced by stimuli as diverse as Fas, TGF- $\beta$, ceramide, and ER stress ${ }^{30-33,36}$. This was also confirmed in the present study in which Dapk1 $1^{-/}$BMDMs and DAPK1knockdown Jurkat $\mathrm{T}$ lymphoma cells were more resistant than their WT counterparts to FasL- or ER stresstriggered death (Supplementary Fig. 5). Furthermore, DAPK1 mediates cell death induced by oxidative stress through phosphorylation of PKD and subsequent activation of $\mathrm{JNK}^{43}$, and during ischemic injury, by activation of the $N$-methyl-d-aspartate (NMDA) glutamate receptor through interaction and phosphorylation of the NR2B subunit $^{46}$. DAPK1 has also been implicated in mediating autophagic cell death ${ }^{36,47,48}$. In contrast, DAPK1deficiency rendered myeloid cells and HT-29 cells more sensitive to necroptosis triggered by treatment with $\mathrm{zVAD}$ plus either SMAC mimetics, TNF, or IFN- $\beta$ (Figs. 1 and 2, Supplementary Figs. 2-4). The ability of DAPK1 to antagonize necroptosis therefore stands out among the capacity of DAPK1 to promote various types of cell death.

DAPK1 targets an early stage of the necroptotic pathway. At least three distinct kinases (IKK $\alpha / \mathrm{IKK} \beta$, TBK1 and MK2) have been shown to directly phosphorylate RIPK1, thereby preventing formation of the RIPK1containing death complex ${ }^{20-25}$. Our findings herein show that DAPK1 regulates inhibitory phosphorylation of RIPK1 at S321 (Fig. 5d). Moreover, DAPK1-deficiency led to diminished TNF-induced p38 MAPK activation, accompanied by reduced MK2 activation and decreased RIPK1 S321 phosphorylation (Fig. 5d). Inhibition of the activation of either p38 MAPK or MK2 conferred the susceptibility to necroptosis induction in WT macrophages (Fig. 5g). Attenuated p38/MK2 activation in Dapk1 $1^{-/-}$BMDMs also increased the sensitivity to necroptosis triggered by SMAC mimetic alone (Fig. 1f). The ability of DAPK1 to inhibit RIPK1 S166 phosphorylation was also demonstrated in the enhanced RIPK1 pS166-mediated apoptosis in Dapk1 ${ }^{-/}$BMDMs
(Supplementary Fig. 10a). Therefore, enhanced formation of the FADD-caspase 8-RIPK1-RIPK3 complex (Fig. 4) is partly attributable to reduced activation of p38 MAK/ MK2 and attenuated phosphorylation of RIPK1 at S321 in DAPK1-deficient cells.

In addition to phosphorylation of the target proteins, DAPK1 has also been shown to regulate target protein function though protein-protein interaction that is independent of DAPK1 catalytic activity. DAPK1 binds and activates pyruvate kinase M2 (PKM2) in the absence of DAPK1 kinase domain ${ }^{49}$. The interaction of DAPK1 death domain with microtubule-affinity regulating kinase (MARK) activates MARK1/2 independent of DAPK1 kinase activity $^{50}$. DAPK1 binds NLRP3 to promote NLRP3 inflammasome activation without the involvement of DAPK1 catalytic activity ${ }^{51}$. In the present study, we also found that the kinase activity of DAPK1 was not essential for repressing necroptosis (Fig. 6b, c). We further found that DAPK1-deficiency specifically attenuated the activation p38 MAPK, but not of ERK and JNK (Fig. 5c-e). In addition, the activation of MKK3 was normal in Dapk1 $1^{-/}$BMDMs (Fig. 6a), suggesting the process regulated by DAPK1 is at the stage of $\mathrm{p} 38$ MAPK. Overexpression of p38 MAPK, but not MKK3, restored the viability of DAPK $1^{-/-}$HT-29 cells to necroptosis induction (Fig. 6d). A possible scaffold role of DAPK1 was suggested by the direct interaction of DAPK1 with p38 MAPK (Fig. 6e, f). We also mapped the cytoskeletonbinding domain of DAPK1 as the region interacting with p38 MAPK (Fig. 6h). Furthermore, in the in vitro kinase analysis containing only p38 MAPK, MKK 3 and ATP, the inclusion of DAPK1 enhanced the phosphorylation of p38 MAPK (Fig. 6g). DAPK1 mutant (DAPK1 $\Delta$ Cyt) that did not bind p38 MAPK failed to enhance p38 MAPK activation in the same assay (Fig. $6 \mathrm{~h}$, i), suggesting that association of p38 MAPK with DAPK is required for the full TNFR-induced p38 MAPK activation. Therefore, our results provided the most direct evidences to illustrate on how DAPK1 increases TNF-directed p38 MAPK activation.

DAPK1 displays anti-inflammatory and proinflammatory activities in different cell types ${ }^{52}$. We previously demonstrated that TCR-induced NF-kB activation is enhanced in Dapk1 $1^{-/-} \mathrm{T}$ cells ${ }^{42,53}$, whereas LPS-triggered NF- $\mathrm{kB}$ activation is modestly reduced in Dapk $1^{-1-} \mathrm{BMDMs}^{51}$. In the present study, TNF-induced NF- $\kappa B$ activation and IKK activation in myeloid cells was not affected by DAPK1-deficiency (Fig. 5c), suggesting that increased necroptosis in Dapk $1^{-1-}$ BMDMs is not linked to the processes of NF- $\mathrm{kB}$ activation. As another example, TCR-induced p38 MAPK activation is normal in Dapk1 $1^{-1-} \mathrm{T}$ cells ${ }^{42}$, while TNFR-initiated p38 MAPK phosphorylation was impaired in Dapk $1^{-/-}$BMDMs (Fig. 5d). These results further support the notion that 
signaling modulation by DAPK1 is cell type- and surface receptor-dependent ${ }^{52}$.

Necroptosis plays a dual role in cancer. Induction of necroptosis is a legitimate approach to killing tumor cells, especially for those that are resistant to apoptotic death, as confirmed by various in vitro studies ${ }^{26,54,55}$. Notably, RIPK1, RIPK3 and/or MLKL are downregulated in various types of cancer ${ }^{56-58}$, reflecting the necessity for tumor cells to circumvent necroptosis ${ }^{26,59}$. Our observation that DAPK1-deficiency increases necroptosis may seem incompatible with DAPK1's tumor-suppressing role. However, our results also suggest that some of DAPK1's inhibitory activity lies at the apex of the necroptotic pathway, i.e., RIPK1 S321 phosphorylation, but many tumors evade necroptosis at the effector stages, e.g. by mutations in RIPK3 or MLKL ${ }^{26,54,55}$. In addition, necroptosis may contribute to tumorigenesis ${ }^{54,59}$. RIPK1RIPK3 necrosomes promote oncogenesis and immune suppression in pancreatic ductal adenocarcinoma ${ }^{60}$. Melanoma cells also trigger necroptosis of endothelial cells to promote tumor extravasation and metastasis ${ }^{61}$. Therefore, the necroptosis-inhibitory activity of DAPK1 does not necessarily contradict its tumor-suppressing function. The application of DAPK1-mediated necroptosis suppression is likely tumor-type and tumor stage dependent. Whether downregulation of DAPK1 in several tumor types may provide an opportunity to target these cancers with necroptosis-inducing agents warrants further exploration.

Necroptosis plays a critical role in the regulation of infection, inflammation and carcinogenesis, and the therapeutic applications of precise necroptosis regulation are well recognized. Recent studies reveal a specific role for RIPK1 phosphorylation at S321/326 in the control of necroptosis ${ }^{21-23}$. Our study suggests the possibility of regulating phosphor-S321/326 RIPK1-mediated necroptosis by modulating DAPK1 levels. Enhanced expression of DAPK1 inhibits necroptosis (Fig. S7), whereas reduced DAPK1 promotes necrosome formation (Figs. 1 and 2). Thus, DAPK1-mediated controlled necroptosis represents a potential therapeutic approach, but future research is needed to identify a reliable means of DAPK1-based necroptotic regulation.

The identification of p38 MAPK-MK2 as target signal molecules by DAPK1 reveals an additional unexpected regulatory pathway. MK2 has been implicated for its tumorigenic role in various cancers ${ }^{62}$. In addition, MK2 directly promotes autoimmune and inflammatory diseases including rheumatoid arthritis, chronic obstructive pulmonary disease, cardiovascular diseases and diabetes $^{63,64}$. The selective association of DAPK1 to p38 MAPK-MK2 activation in macrophages provides a prospect in the regulation of MK2 activation and MK2associated inflammatory pathology. Whether DAPK1 downregulation could be used to treat diseases mediated by $\mathrm{p} 38-\mathrm{MK} 2$ over-activation also deserves further investigation.

\section{Materials and methods \\ DAPK1 expression and knockout}

Human DAPK1-specific siRNA (siDAPK1; UCU GGG AAG CGG AGC UGA AUU) and siRNA control (siCtrl) were purchased from GE Dharmacon (Lafayette, CO, USA). Dapk $1^{-1-}$ mice (in a C57BL/6 background) were previously described ${ }^{36}$. Mice were maintained in the SPF mouse facility of the Institute of Molecular Biology, Academia Sinica. All mouse experiments were conducted with approval from the Institutional Animal Care \& Utilization Committee, Academia Sinica.

All mice used in this study were 8-12-week old. The same sex (male or female) mice were used in the same experiment, but opposite sex mice could be used in the repeat of the given experiment. No difference was observed between male and female mice in the analyses conducted in this study. Experimental groups were assigned randomly. Five or more mice in each experimental group was planned, but four mice in some experimental groups, that have been examined in previous studies, were used due to the knockout-mice availability. No blinding was done because the readouts of the mouse experiments in this study were clear-cut (body weight loss, death). No mice were excluded from scoring.

\section{Reagents}

Purified LPS was purchased from InvivoGen (San Diego, CA). AT-406 was synthesized as described ${ }^{65}$. Flag-M2 antibodies, Flag-M2 beads, necrostatin-1 (Nec-1), cycloheximide (CHX), thapsigargin (THAP), propidium iodide (PI) and (3-(4,5-dimethylthiazol-2-yl)-2,5-diphenyl tetrazolium bromide) (MTT) were purchased from Sigma (St. Louis, MO). JNK inhibitor II (SP600125) and DMSO were purchased from Merck Millipore (Billerica, MA). Annexin V-Cy5 was obtained from BD-Biosciences (Franklin Lakes, NJ). Recombinant GM-CSF was purchased from R\&D (Minneapolis, MN, USA). z-VAD-FMK was obtained from Bachem (Bubendorf, Switzerland). Recombinant mouse and human TNF were purchased from PeproTech (Rocky Hill, NJ, USA). Recombinant mouse IFN $\beta$ was purchased from PBL Assay Science (Piscataway, NJ, USA). BV6 was purchased from Selleck Chemicals (Houston, TX). Recombinant human Fas ligand (FLAG-FasL) was purchased from Enzo Life Sciences (Farmingdale, NY, USA). CellTiter-Glo ${ }^{\circledR}$ Luminescent Cell Viability Assay was purchased from Promega (Fitchburg, Wisconsin). Lipofectamine 2000 was purchased from Thermo Fisher Scientific (Waltham, MA, USA). Constructs of $\mathrm{p} 38 \alpha$ MAPK and active MKK3 
$\left(\mathrm{MKK} 3 \mathrm{~b}\left(\mathrm{Glu}^{189}, \mathrm{Glu}^{193}\right)\right)$ were gifts of Dr. Jiahuai Han (Xiamen University, China).

\section{Antibodies}

Anti-DAPK1 (D2178, DAPK-55, Western blot (WB) 1:1000), anti-HA (H3633, HA-7, WB 1:1000, immunoprecipitation (IP) $1 \mu \mathrm{g}$ per test) and anti-FLAG (F1804, M2, WB 1:1000, IP $1 \mu \mathrm{g}$ per test) were purchased from Sigma (St. Louis, MO). Anti-cIAP2 (sc-7944, H-85, WB 1:1000), anti-human FADD (sc-5559, H-181, IP 1:500), anti-mouse FADD (sc-6036, M-19, IP 1:500), anti-human caspase-8 (sc-6136, C-20, IP 1:500), anti-AKT (sc-5298, B1, WB 1:1000), anti-ERK (sc-154, C-14, WB 1:4000), antip38 MAPK (sc-535-G, C-20, WB 1:1000), anti-IkBa (sc371, C-21, WB 1:2000) and anti-GAPDH (sc-32233, 6C5, WB 1:10000) were all purchased from Santa Cruz Biotech (Santa Cruz, CA). Anti-cIAP1 (GTX110087, WB 1:6000) was purchased from GeneTex (Irving, CA). Anti-human phospho RIPK1 ${ }^{\text {S166 }}$ (65746, D1L3S, WB 1:1000), antimouse phospho RIPK1 ${ }^{\mathrm{S} 166}$ (31122, WB 1:1000), antihuman RIPK3 (13526, E1Z1D, WB 1:4000), anti-mouse RIPK3 (95702, D4G2A, WB 1:6000), anti-human caspase8 (9746, 1C12, WB 1:500), anti-mouse caspase-8 (4927, WB 1:1000), anti-phospho AKT ${ }^{\mathrm{S} 473}$ (9271, WB 1:1000), anti-JNK (9252, WB 1:1000), anti-phospho JNK ${ }^{\mathrm{T} 183 / \mathrm{Y} 185}$ (9251, WB 1:1000), anti-phospho ERK ${ }^{\mathrm{T} 202 / \mathrm{Y} 204}$ (9101, WB 1:4000), anti-phospho $\mathrm{I}_{\kappa} B \alpha^{\mathrm{S} 32 / \mathrm{S} 36}$ (9246, 5A5, WB 1:1500), anti-phospho IKK $\alpha^{\text {S176 }} / \mathrm{IKK} \beta^{\text {S177 }}$ (2078, C84E11, WB 1:1000), anti-phospho TBK1 $1^{\mathrm{S} 172}$ (5483, D52C2, WB 1:1000), anti-phospho RIPK1 ${ }^{\mathrm{S} 321}$ (83613, WB 1:2000), anti-phospho $\mathrm{p} 38^{\mathrm{T} 180 / \mathrm{Y} 182}$ (9211, WB 1:1000), antiphospho MK2 ${ }^{\mathrm{T} 334}$ (3007, WB 1:500), anti-IKK $\beta$ (8943, D30C6, WB 1:1000), anti-TBK1 (3504, WB 1:1000), antiMK2 (3042, WB 1:1000) and anti-Myc (2276, 9B11, WB 1:1000, IP $1 \mu \mathrm{g}$ per test) were all purchased from Cell Signaling Technology (Danvers, MA). Anti-mouse RIPK3 (2283, WB 1:6000) was purchased from ProSci (San Diego, CA). Anti-human phospho RIPK3 ${ }^{\mathrm{S} 227}$ (ab209384, EPR9627, WB 1:4000), anti-mouse phospho RIPK3 ${ }^{\text {S232 }}$ (ab195117, EPR9516(N)-25, WB 1:3000), anti-phospho p38 ${ }^{\mathrm{T} 180 / Y 182}$ (ab170099), anti-human MLKL (ab184718, EPR17514, WB 1:6000), anti-human phospho MLKL ${ }^{\mathrm{S} 358}$ (ab187091, EPR9514, WB 1:6000), anti-mouse phospho MLKL $^{\text {S345 }}$ (ab196436, EPR9515(2), WB 1:6000) and antimouse caspase-8 (ab138485, IP 1:500) antibodies were purchased from Abcam (Cambridge, UK). Anti-FADD (05-486, 1F7, WB 1:1000) and anti-mouse MLKL (MABC604, 3H1, WB 1:2000) were purchased from Merck Millipore (Billerica, MA). Anti-RIPK1 (610459, 38/ RIP, WB 1:6000), anti-HSP90 (610419, 68/Hsp90, WB 1:4000), anti-CD3-PE (145-2C11, flow cytometry (FCM) 1:100), anti-Gr-1-PE (RB6-8C5, FCM 1:100) and anti-IAb-PE (AF6-120.1, FCM 1:100) were obtained from BDBiosciences (Franklin Lakes, NJ). Anti-CD4-PE (RM4-4,
FCM 1:100), anti-B220-FITC (RA3-6B2, FCM 1:100), anti-CD11b-PE-Cy7 (M1/70, FCM 1:100), anti-CD11c-PB (N418, FCM 1:100), anti-F4/80-APC (BM8, FCM 1:100) and anti-Fas-APC (DX2, 1:100) antibodies were purchased from BioLegend (San Diego, CA, USA). Anti-CD8PE (53-6.7, FCM 1:100) and anti-Ly6C-APC (HK1.4, FCM 1:100) were purchased from eBioscience (San Diego, CA, USA).

\section{Cell culture}

HEK293T (ATCC CRL-3216), HT-29 (ATCC HTB-38), J774A.1 (ATCC TIB-67) and Jurkat T lymphoma (clone E6-1, ATCC TIB-152) and L929 (ATCC CCL-1) cell lines were obtained from ATCC. Cell lines were examined for mycoplasma contamination using a Mycoplasma Detection Kit (R\&D). HT-29 cells and Jurkat cells were cultured in complete RPMI-1640 medium containing $10 \%$ fetal calf serum (Invitrogen Life Technology), $10 \mathrm{mM}$ glutamine, $100 \mathrm{U} / \mathrm{ml}$ penicillin, $100 \mu \mathrm{g} / \mathrm{ml}$ streptomycin and $50 \mu \mathrm{M}$ 2-mercaptoethanol. HEK293T, J774A.1 and L929 cells were cultured in complete DMEM medium with the same supplements as for complete RPMI medium. Bone marrow cells were collected from tibias and femurs by flushing with cold phosphate buffered saline (PBS). Bone marrow cells were cultured in complete DMEM medium with 20\% L929-conditioned media for 6 days to generate bone marrow-derived macrophages (BMDMs). Bone marrow cells were also differentiated in complete RPMI medium containing $20 \mathrm{ng} / \mathrm{ml} \mathrm{GM-CSF}$ for 8 days to generate bone marrow-derived dendritic cells (BMDCs). DAPK was also knocked down in HT-29 cells by transfection with siDAPK1 using Lipofectamine 2000.

\section{Cell viability assay}

Necroptosis was induced by pretreating cells with z-VAD for $0.5 \mathrm{~h}$, followed by stimulation with AT406, BV6, LPS, TNF or IFN $\beta$ for the indicated periods. The necroptosis inhibitor Nec-1 was added $0.5 \mathrm{~h}$ prior to stimulation for certain experiments. Cell viability of BMDMs or BMDCs was assessed by measuring ATP levels upon adding an equal volume of Cell Titer-Glo reagent (Promega) and incubating for $30 \mathrm{~min}$. Luminescence was determined using a Victor3 1420 Multilabel Counter (PerkinElmer, Shelton, CT). Alternatively, cell viability was determined via reduction of MTT by mitochondrial reductase into purple formazan. The intensity of colored product was measured by absorbance at $490 \mathrm{~nm}$ on an Emax microtiter plate reader (Molecular Device, Sunnyvale, CA). Necroptotic HT-29 cells were determined by staining with PI $(10 \mu \mathrm{g} / \mathrm{ml})$ in PBS, and $\mathrm{PI}^{+}$cells were analyzed using flow cytometry. Apoptosis of Jurkat cells was induced by treating with FLAG-FasL and assessed by staining with Annexin $\mathrm{V}-\mathrm{Cy} 5$ (BD-Biosciences) and subsequent Annexin $\mathrm{V}^{+}$cell quantitation by flow cytometry. 


\section{Generation of DAPK1 knockout HT-29 cell by CRISPR-Cas9 editing}

$D A P K 1^{-1-}$ HT-29 cells were generated using an All-inOne Cas $9^{\text {D10A }}$ nickase system ${ }^{66}$. AIO-GFP plasmid was purchased from Addgene (\#74119). The specific DAPK1sgRNA was designed using CRISPR Design (http://crispr. mit.edu/) at exon 2 of the DAPK1 locus on chromosome 9. A pair of sense DAPK1-sgRNA was annealed using $5^{\prime}$ -accgTGA TTA CTA CGA CAC CGG CG-3' and 5'aaacCG CCG GTG TCG TAG TAA TCA-3', and a pair of antisense DAPK-sgRNA was annealed using $5^{\prime}$-accgCAC GTT TTC CTG CCT GAA CA-3' and 5'-aaacTG TTC AGG CAG GAA AAC GTG-3' (Supplementary Fig. 5a). AIO-GFP plasmid containing DAPK1-sgRNA was transfected into HT-29 cells by electroporation using the MP100 system (Life Technologies), and $\mathrm{GFP}^{+}$cells were isolated by cell sorting. Sorted cells were sub-cultured into 96-well plates for single clone selection. Exon 2 of the $D A P K 1$ locus was amplified from individual clones by PCR and sequenced to verify its deletion.

\section{Western blot and immunoprecipitation}

For immunoblotting and immunoprecipitation, cells were lysed by whole-cell extract (WCE) buffer $(25 \mathrm{mM}$ HEPES pH 7.9, $300 \mathrm{mM} \mathrm{NaCl}, 1.5 \mathrm{mM} \mathrm{MgCl}_{2}, 0.2 \mathrm{mM}$ EDTA, $0.5 \mathrm{mM}$ DTT and $0.1 \%$ Triton X-100) on ice for $30 \mathrm{~min}$. Supernatants were separated by centrifugation at $13,200 \mathrm{rpm}$ for $10 \mathrm{~min}$ at $4{ }^{\circ} \mathrm{C}$. The protein concentrations were determined by Bio-Rad protein assay (\#500-00006). For immunoprecipitation, $0.5 \mathrm{mg}$ total cell lysates were incubated with $1 \mu \mathrm{g}$ specific antibody, and the mixtures were rotated overnight at $4{ }^{\circ} \mathrm{C}$. The immune-complexes were captured by Protein G Mag Sepharose (GE, 28-9670$70,10 \mu$ l per sample). The beads were washed three times with WCE buffer and denatured by 4X SDS sample buffer (200 mM Tris- $\mathrm{HCl}$ pH 6.8, 1.2 M $\beta$-mercaptoethanol, 40\% glycerol, $8 \%$ SDS, and $0.4 \%$ bromophenol blue). For immunoblots, samples were denatured and analyzed by SDS-PAGE with running buffer (0.025 M Tris, $0.192 \mathrm{M}$ glycine, and $0.1 \%$ SDS). The PAGE was transferred to PVDF membrane (Millipore) with transfer buffer (25 mM Tris, $192 \mathrm{mM}$ glycine, and 20\% methanol) at $400 \mathrm{~mA}$ for $100 \mathrm{~min}$ at $4{ }^{\circ} \mathrm{C}$. Membranes were blocked with SuperBlock $^{\mathrm{TM}}$ T20 (Sigma) to detect anti-phosphate antibody or blocking buffer ( $5 \%$ non-fat milk and $0.1 \%$ Tween-20 in a TBST buffer of $50 \mathrm{mM}$ Tris- $\mathrm{HCl} \mathrm{pH} 7.4$ and $150 \mathrm{mM}$ $\mathrm{NaCl}$ ) at room temperature for $30 \mathrm{~min}$, before being incubated with specific primary antibodies at indicated dilutions at $4{ }^{\circ} \mathrm{C}$ overnight. The membranes were washed three times with wash buffer $(0.1 \%$ Tween-20 in TBST buffer) at room temperature for $10 \mathrm{~min}$ before incubating them with horseradish peroxidase-conjugated secondary antibodies in blocking buffer at room temperature for $1 \mathrm{~h}$. After washing, the membranes were developed with ECL
Western blot detection reagents (Advansta, K-12045D50), with signals detected by X-ray film (Fujifilm).

\section{TNF-induced septic shock}

Wild-type littermates or Dapk1-knockout C57BL/6 mice of 6-8 weeks and same sex were used for TNFinduced septic shock. No randomization was used due to the availability of knockout mice. Mice were anesthetized by Avertin $(0.25 \mathrm{ml}$ of $20 \mathrm{mg} / \mathrm{ml}$, Sigma) and mouse TNF $(1.0 \mu \mathrm{g} / \mathrm{g})$ was intravenously administered in total volume of $200 \mu \mathrm{l}$ endotoxin-free PBS. Body temperatures were monitored from rectal by industrial electric thermometer (Kane-may) for $30 \mathrm{~h}$, with simultaneous recording of mice death. Mice were sacrificed when body temperature fell below $22^{\circ} \mathrm{C}$. No blinding was done because the readouts of septic shock were straightforward (temperature drop, death). No mice were excluded from scoring.

\section{In vitro kinase analysis}

Recombinant human His-p38 MAPK (ab82188) and recombinant human His-MKK3 (ab105578) were obtained from Abcam. DAPK-FLAG protein was purified from cell lysates of HEK293T cells transfected with DAPK-FLAG, by anti-FLAG M2 affinity gel and eluted with $3 \times$ FLAG peptides. Recombinant p38 MAPK (50 ng) and recombinant MKK3 (100 ng) were incubated with purified DAPK-FLAG $(0,25 \mathrm{ng}$ or $75 \mathrm{ng})$ in kinase buffer (200 $\mu \mathrm{M}$ ATP, $20 \mathrm{mM}$ HEPES (pH 7.6), $20 \mathrm{mM} \mathrm{MgCl}_{2}$, $1 \mathrm{mM} \mathrm{DTT}$ ) at $30^{\circ} \mathrm{C}$ for $1 \mathrm{~h}$. The extent of p38 MAPK phosphorylation was determined.

\section{Statistics}

Data were randomly collected, but not blindly. We did not exclude any data from this study. Data met the assumptions of applied statistical tests (i.e. normal distributions). Microsoft Office Excel and Prism 5.0 (GraphPad software) were used for data analysis. Unpaired two-tailed Student's $t$ tests were used to compare most of the viability or death results between two groups. Two-way ANOVA followed by a Tukey's multiple comparison tests, or two-way ANOVA followed by a Sidak's multiple comparison test were used to compare other results, as indicated in the respective figure legends. Long-rank (Mantel-Cox) test was used to compare the survival of mice. Data are presented as means with standard deviation (SD).

\section{Acknowledgements}

The authors thank Yamin Lin and staff of the FACS Core of the Institute of Molecular Biology Academia Sinica (IMB) for cell sorting, Sue-Ping Lee and staff of the Confocal Core of IMB for confocal microscopy, and Dr. John O'Brien for editing the manuscript. This work was supported by grant MOST107-2321-B001-031 from the Ministry of Science and Technology, and with additional support from Academia Sinica, Taiwan, R.O.C. 


\section{Author details}

'Institute of Molecular Biology, Academia Sinica, Taipei 11529, Taiwan. 2Department of Molecular Genetics, Weizmann Institute of Science, Rehovot 76100, Israel. ${ }^{3}$ Institute of Biological Chemistry, Academia Sinica, Taipei 11529, Taiwan

\section{Conflict of interest}

The authors declare that they have no conflict of interest.

\section{Publisher's note}

Springer Nature remains neutral with regard to jurisdictional claims in published maps and institutional affiliations.

Supplementary Information accompanies this paper at (https://doi.org/ 10.1038/s41419-020-2534-9).

Received: 16 December 2019 Revised: 17 April 2020 Accepted: 20 April 2020

Published online: 04 May 2020

\section{References}

1. Galluzzi, L. et al. Molecular mechanisms of cell death: recommendations of the Nomenclature Committee on Cell Death 2018. Cell Death Differ. 25, 486-541 (2018).

2. Annibaldi, A. \& Meier, P. Checkpoints in TNF-Induced Cell Death: Implications in Inflammation and Cancer. Trends Mol. Med. 24, 49-65 (2018).

3. Pasparakis, M. \& Vandenabeele, P. Necroptosis and its role in inflammation. Nature 517, 311-320 (2015).

4. Chan, F. K., Luz, N. F. \& Moriwaki, K. Programmed necrosis in the cross talk of cell death and inflammation. Annu. Rev. Immunol. 33, 79-106 (2015).

5. Wallach, D., Kang, T. B., Dillon, C. P. \& Green, D. R. Programmed necrosis in inflammation: toward identification of the effector molecules. Science $\mathbf{3 5 2}$, aaf2154 (2016).

6. Weinlich, R., Oberst, A., Beere, H. M. \& Green, D. R. Necroptosis in development, inflammation and disease. Nat. Rev. Mol. Cell Biol. 18, 127-136 (2017).

7. Robinson, N. et al. Type I interferon induces necroptosis in macrophages during infection with Salmonella enterica serovar Typhimurium. Nat. Immunol. 13, 954-962 (2012).

8. Micheau, O. \& Tschopp, J. Induction of TNF receptor I-mediated apoptosis via two sequential signaling complexes. Cell 114, 181-190 (2003).

9. He, S. et al. Receptor interacting protein kinase-3 determines cellular necrotic response to TNF-alpha. Cell 137, 1100-1111 (2009).

10. Cho, Y. S. et al. Phosphorylation-driven assembly of the RIP1-RIP3 complex regulates programmed necrosis and virus-induced inflammation. Cell 137, 1112-1123 (2009)

11. Zhang, D. W. et al. RIP3, an energy metabolism regulator that switches TNF-induced cell death from apoptosis to necrosis. Science $\mathbf{3 2 5}$ 332-336 (2009)

12. Sun, $L$. et al. Mixed lineage kinase domain-like protein mediates necrosis signaling downstream of RIP3 kinase. Cell 148, 213-227 (2012).

13. Zhao, J. et al. Mixed lineage kinase domain-like is a key receptor interacting protein 3 downstream component of TNF-induced necrosis. Proc. Natl Acad. Sci. USA 109, 5322-5327 (2012).

14. Wang, $\mathrm{H}$. et al. Mixed lineage kinase domain-like protein MLKL causes necrotic membrane disruption upon phosphorylation by RIP3. Mol. Cell 54, 133-146 (2014).

15. Cai, Z. et al. Plasma membrane translocation of trimerized MLKL protein is required for TNF-induced necroptosis. Nat. Cell Biol. 16, 55-65 (2014).

16. Chen, $X$. et al. Translocation of mixed lineage kinase domain-like protein to plasma membrane leads to necrotic cell death. Cell Res. 24, 105-121 (2014).

17. Kaiser, W. J. et al. RIP3 mediates the embryonic lethality of caspase-8-deficient mice. Nature 471, 368-372 (2011).

18. Zhang, $\mathrm{H}$. et al. Functional complementation between FADD and RIP1 in embryos and lymphocytes. Nature 471, 373-376 (2011).

19. Oberst, A. et al. Catalytic activity of the caspase-8-FLIP(L) complex inhibits RIPK3-dependent necrosis. Nature 471, 363-367 (2011).
20. Dondelinger, Y. et al. NF-kappaB-independent role of IKKalpha/IKKbeta in preventing RIPK1 kinase-dependent apoptotic and necroptotic cell death during TNF signaling. Mol. Cell 60, 63-76 (2015)

21. Jaco, I. et al. MK2 phosphorylates RIPK1 to prevent TNF-induced cell death Mol. Cell 66, 698-710 e695 (2017).

22. Menon, M. B. et al. p38(MAPK)/MK2-dependent phosphorylation controls cytotoxic RIPK1 signalling in inflammation and infection. Nat. Cell Biol. 19, 1248-1259 (2017).

23. Dondelinger, Y. et al. MK2 phosphorylation of RIPK1 regulates TNF-mediated cell death. Nat. Cell Biol. 19, 1237-1247 (2017).

24. $\mathrm{XU}$, D. et al. TBK1 suppresses RIPK1-driven apoptosis and inflammation during development and in aging. Cell 174, 1477-1491 e1419 (2018).

25. Lafont, E. et al. TBK1 and IKKepsilon prevent TNF-induced cell death by RIPK1 phosphorylation. Nat. Cell Biol. 20, 1389-1399 (2018)

26. Galluzzi, L, Kepp, O, Chan, F. K. \& Kroemer, G. Necroptosis: mechanisms and relevance to disease. Annu Rev. Pathol. 12, 103-130 (2017).

27. Shiloh, R., Bialik, S. \& Kimchi, A. The DAPK family: a structure-function analysis. Apoptosis 19, 286-297 (2014)

28. Farag, A. K. \& Roh, E. J. Death-associated protein kinase (DAPK) family modulators: current and future therapeutic outcomes. Med. Res. Rev. 39, 349-385 (2019).

29. Bialik, S. \& Kimchi, A. The death-associated protein kinases: structure, function, and beyond. Annu. Rev. Biochem. 75, 189-210 (2006)

30. Deiss, L. P., Feinstein, E, Berissi, H., Cohen, O \& Kimchi, A. Identification of a novel serine/threonine kinase and a novel $15-\mathrm{kD}$ protein as potential mediators of the gamma interferon-induced cell death. Genes Dev 9, 15-30 (1995).

31. Cohen, O. et al. DAP-kinase participates in TNF-alpha- and Fas-induced apoptosis and its function requires the death domain. J. Cell Biol. 146, 141-148 (1999).

32. Jang, C. W. et al. TGF-beta induces apoptosis through Smad-mediated expression of DAP-kinase. Nat. Cell Biol. 4, 51-58 (2002).

33. Pelled, D. et al. Death-associated protein (DAP) kinase plays a central role in ceramide-induced apoptosis in cultured hippocampal neurons. J. Biol. Chem. 277, 1957-1961 (2002).

34. Wang, W. J., Kuo, J. C., Yao, C. C. \& Chen, R. H. DAP-kinase induces apoptosis by suppressing integrin activity and disrupting matrix survival signals. J. Cell Biol. 159, 169-179 (2002).

35. Llambi, F. et al. The dependence receptor UNC5H2 mediates apoptosis through DAP-kinase. EMBO J. 24, 1192-1201 (2005).

36. Gozuacik, D. et al. DAP-kinase is a mediator of endoplasmic reticulum stressinduced caspase activation and autophagic cell death. Cell Death Differ. 15 1875-1886 (2008)

37. Bialik, S. \& Kimchi, A. The DAP-kinase interactome. Apoptosis 19, 316-328 (2014).

38. Henshall, D. C. et al. Expression of death-associated protein kinase and recruitment to the tumor necrosis factor signaling pathway following brief seizures. J. Neurochem. 86, 1260-1270 (2003).

39. Raveh, T., Droguett, G., Horwitz, M. S., DePinho, R. A. \& Kimchi, A. DAP kinase activates a p19ARF/p53-mediated apoptotic checkpoint to suppress oncogenic transformation. Nat. Cell Biol. 3, 1-7 (2001).

40. Gozuacik, D. \& Kimchi, A. DAPk protein family and cancer. Autophagy 2, 74-79 (2006).

41. Michie, A. M., McCaig, A. M., Nakagawa, R. \& Vukovic, M. Death-associated protein kinase (DAPK) and signal transduction: regulation in cancer. FEBS $\mathrm{J}$. 277, 74-80 (2010).

42. Chuang, Y. T., Fang, L. W., Lin-Feng, M. H., Chen, R. H. \& Lai, M. Z. The tumor suppressor death-associated protein kinase targets to TCR-stimulated NFkappa B activation. J. Immunol. 180, 3238-3249 (2008).

43. Eisenberg-Lerner, A. \& Kimchi, A. DAP kinase regulates JNK signaling by binding and activating protein kinase D under oxidative stress. Cell Death Differ. 14, 1908-1915 (2007).

44. Ben-Levy, R., Hooper, S, Wilson, R, Paterson, H. F. \& Marshall, C. J. Nuclear export of the stress-activated protein kinase p38 mediated by its substrate MAPKAP kinase-2. Curr. Biol. CB 8, 1049-1057 (1998).

45. Engel, K., Kotlyarov, A. \& Gaestel, M. Leptomycin B-sensitive nuclear export of MAPKAP kinase 2 is regulated by phosphorylation. Embo J. 17, 3363-3371 (1998).

46. Tu, W. et al. DAPK1 interaction with NMDA receptor NR2B subunits mediates brain damage in stroke. Cell 140, 222-234 (2010).

47. Levin-Salomon, V., Bialik, S. \& Kimchi, A. DAP-kinase and autophagy. Apoptosis 19, 346-356 (2014) 
48. Inbal, B., Bialik, S., Sabanay, I., Shani, G. \& Kimchi, A. DAP kinase and DRP-1 mediate membrane blebbing and the formation of autophagic vesicles during programmed cell death. J. Cell Biol. 157, 455-468 (2002).

49. Mor, I., Carlessi, R., Ast, T., Feinstein, E. \& Kimchi, A. Death-associated protein kinase increases glycolytic rate through binding and activation of pyruvate kinase. Oncogene 31, 683-693 (2012).

50. Wu, P. R. et al. DAPK activates MARK1/2 to regulate microtubule assembly, neuronal differentiation, and tau toxicity. Cell Death Differ. 18, 1507-1520 (2011).

51. Chuang, Y. T. et al. Tumor suppressor death-associated protein kinase is required for full IL-1beta production. Blood 117, 960-970 (2011).

52. Lai, M. Z. \& Chen, R. H. Regulation of inflammation by DAPK. Apoptosis 19 357-363 (2014).

53. Chou, T. F. et al. Tumour suppressor death-associated protein kinase targets cytoplasmic HIF-1alpha for Th17 suppression. Nat. Commun. 7, 11904 (2016).

54. Wang, T. et al. Necroptosis in cancer: an angel or a demon? Tumour Biol. 39, 1010428317711539 (2017).

55. Su, Z., Yang, Z., Xie, L., DeWitt, J. P. \& Chen, Y. Cancer therapy in the necroptosis era. Cell Death Differ. 23, 748-756 (2016).

56. Feng, $\mathbf{X}$. et al. Receptor-interacting protein kinase 3 is a predictor of survival and plays a tumor suppressive role in colorectal cancer. Neoplasma $6 \mathbf{2}$ 592-601 (2015).

57. Nugues, A. L. et al. RIP3 is downregulated in human myeloid leukemia cells and modulates apoptosis and caspase-mediated p65/RelA cleavage. Cell Death Dis. 5, e1384 (2014).
58. Koo, G. B. et al. Methylation-dependent loss of RIP3 expression in cancer represses programmed necrosis in response to chemotherapeutics. Cell Res. 25, 707-725 (2015).

59. Najafov, A., Chen, H. \& Yuan, J. Necroptosis and cancer. Trends Cancer 3, 294-301 (2017)

60. Seifert, L. et al. The necrosome promotes pancreatic oncogenesis via CXCL1 and Mincle-induced immune suppression. Nature 532, 245-249 (2016).

61. Strilic, B. et al. Tumour-cell-induced endothelial cell necroptosis via death receptor 6 promotes metastasis. Nature 536, 215-218 (2016)

62. Menon, M. B. \& Gaestel, M. MK2-TNF-signaling comes full circle. Trends Biochem. Sci. 43, 170-179 (2018).

63. Ruiz, M., Coderre, L., Allen, B. G. \& Des Rosiers, C. Protecting the heart through MK2 modulation, toward a role in diabetic cardiomyopathy and lipid metabolism. Biochim. Biophysica Acta (BBA)-Mol. Basis Dis. 1864, 1914-1922 (2018)

64. Singh, R. K., Najmi, A. K. \& Dastidar, S. G. Biological functions and role of mitogen-activated protein kinase activated protein kinase 2 (MK2) in inflammatory diseases. Pharm. Rep. 69, 746-756 (2017).

65. Cai, Q. et al. A potent and orally active antagonist (SM-406/AT-406) of multiple inhibitor of apoptosis proteins (IAPS) in clinical development for cancer treatment. J. Med. Chem. 54, 2714-2726 (2011).

66. Chiang, T. W., le Sage, C., Larrieu, D., Demir, M. \& Jackson, S. P. CRISPR-Cas 9 (D10A) nickase-based genotypic and phenotypic screening to enhance genome editing. Sci. Rep. 6, 24356 (2016). 